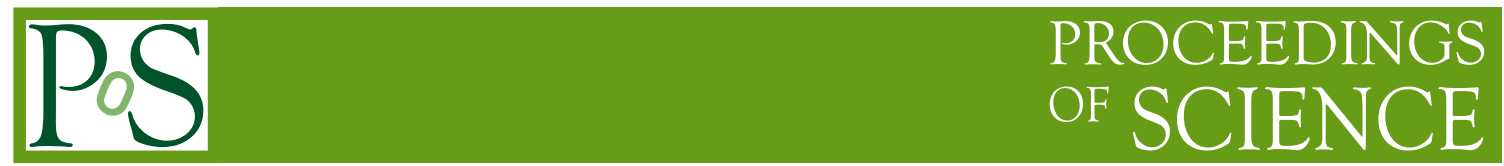

\title{
Chiral Perturbation Theory with a scalar field
}

\author{
Jaume Tarrús Castellà* \\ Departament d'Estructura i Constituents de la Matèria and Institut de Ciències del Cosmos \\ E-mail: tarrusdecm.ub.edu
}

\begin{abstract}
We report on an extension of the usual chiral perturbation theory framework $(\chi \mathrm{PT})$ to allow the inclusion of a light dynamical isosinglet scalar. We present the results for the pion mass an decay constant up to NLO and the S-wave pion-pion scattering lengths at LO in this framework. Using lattice QCD results, and a few phenomenological inputs, we explore the parameter space of the effective theory. We also show how to extract the mass and width of the sigma resonance from chiral extrapolations of lattice QCD data.
\end{abstract}

The 7th International Workshop on Chiral Dynamics,

August 6 -10, 2012

Jefferson Lab, Newport News, Virginia, USA

${ }^{*}$ Speaker. 


\section{Introduction}

Chiral Perturbation Theory, $\chi$ PT [1], has become a standard tool for the phenomenological description of QCD processes involving pseudo-Goldstone bosons at low-energy. However, when pion scattering amplitudes are calculated in the isoscalar channel, a bad convergence is observed, even at reasonably low-momenta. This has led some authors to resum certain classes of diagrams, using a number of unitarization techniques (see, for instance, [2, 3]). Most of these approaches improve considerably the description of data with respect to standard $\chi \mathrm{PT}$, and indicate that a scalar isospin zero resonance at relatively low-mass, the sigma, exist. In fact the mass and width of the sigma resonance are nowadays claimed to be known very accurately $m_{\sigma}=441_{-8}^{+16} \mathrm{MeV}, \Gamma / 2=$ $272_{-12.5}^{+9} \mathrm{MeV}$ [4] (see also [5]). The relatively low-mass of the sigma resonance, with respect to the chiral cutoff and its proximity to the value of the kaon mass suggests that it may be convenient to introduce it as an explicit degree of freedom in an extension of $\chi$ PT. We implement this observation here in a chiral effective theory framework that involves a dynamical singlet field together with the lowest pseudo-Goldstone bosons.

\section{Lagrangian and power counting}

Our aim is to construct an effective field theory containing pions and a singlet scalar field as a degrees of freedom, that holds for processes involving only low-energy pions as the asymptotic states

$$
p, m_{\pi}(\sim 140 \mathrm{MeV}), m_{S}(\sim 440 \mathrm{MeV}) \ll \Lambda_{\chi} .
$$

being $p$ a typical momentum. More refined hierarchies, like $m_{\pi} \ll m_{S}, p \ll \Lambda_{\chi}$ may be interesting to explore in the future.

Consider first the sector containing only the singlet scalar field. In the absence of any symmetry hint we are forced to write the most general polynomial functional,

$$
\mathscr{L}^{S}=\frac{1}{2} \partial_{\mu} S \partial^{\mu} S-\frac{1}{2} \stackrel{\circ}{S}_{S}^{2} S S-\lambda_{1} S-\frac{\lambda_{3}}{3 !} S^{3}-\frac{\lambda_{4}}{4 !} S^{4}+\cdots
$$

where the dots indicate terms suppressed by powers of $1 / \Lambda_{\chi}$. At LO $\lambda_{1}$ must be set to zero in order to avoid mixing of $S$ with the vacuum, and at higher orders it must be adjusted for the same purpose. Their natural sizes for $\lambda_{3}$ and $\lambda_{4}$ are $\lambda_{3} \sim \mathscr{O}\left(\Lambda_{\chi}\right)$ and $\lambda_{4} \sim \mathscr{O}(1)$. In that case, the scalar sector becomes strongly coupled. However, strongly coupled scalar theories in four dimensions are believed to be trivial $[8,9]$. A practical way of taking this fact into account is just setting $\lambda_{3}=\lambda_{4}=0$, which we will do in the following. When the interactions of the scalar with the pseudo-Goldstone bosons are taken into account, small $\left(\stackrel{\circ}{m}_{S}^{2} / \Lambda_{\chi}^{2}\right.$ suppressed) but non-vanishing values of $\lambda_{3}$ and $\lambda_{4}$ are required to ensure perturbative renormalization.

The second contribution we are interested in is the lowest order Lagrangian describing the interaction of the scalar field with the pseudo-Goldstone bosons.

$$
\mathscr{L}^{(2)}=\left(\frac{F^{2}}{4}+F c_{1 d} S+c_{2 d} S^{2}+\cdots\right)\left\langle D_{\mu} U D^{\mu} U^{\dagger}\right\rangle+\left(\frac{F^{2}}{4}+F c_{1 m} S+c_{2 m} S^{2}+\cdots\right)\left\langle\chi^{\dagger} U+\chi U^{\dagger}\right\rangle
$$

where the ellipsis stand for higher order terms involving more powers of the singlet field (or derivatives on them), which are suppressed by powers of $1 / \Lambda_{\chi}$. In computing loop graphs, we will 
encounter divergences. The counter-terms we will need to eliminate the divergences are

$$
\begin{aligned}
& \mathscr{L}^{(4)}=\frac{\ell_{3}}{16}\left\langle\chi^{\dagger} U+\chi U^{\dagger}\right\rangle^{2}+\frac{\ell_{4}}{4}\left\langle D^{\mu} U^{\dagger} D_{\mu} \chi+D^{\mu} \chi^{\dagger} D_{\mu} U\right\rangle+Z_{1} \stackrel{\circ}{m}_{S}^{2}\left\langle\chi^{\dagger} U+\chi U^{\dagger}\right\rangle+Z_{2} \stackrel{\circ}{m}_{S}^{2}\left\langle D_{\mu} U D^{\mu} U^{\dagger}\right\rangle \\
& +f_{2 p} \square S \square S+d_{2 m} \partial_{\mu} S \partial^{\mu} S\left\langle\chi^{\dagger} U+\chi U^{\dagger}\right\rangle+b_{2 m} S^{2}\left\langle\chi^{\dagger} U+\chi U^{\dagger}\right\rangle^{2}+a_{2 m} S^{2}\left\langle\chi^{\dagger} \chi\right\rangle+e_{2 m} S^{2} \Re[\operatorname{det}(\chi)] .
\end{aligned}
$$

Note that $B, F$ and $\ell_{i}$ are equivalent to the corresponding $\chi \mathrm{PT}$ low-energy constants, but they do not need to take same values as in $\chi$ PT.

\subsection{Chiral symmetry constraints}

To envisage the effects of explicit chiral symmetry breaking on the dynamics of the singlet field we set $U$ to the vacuum configuration $(U=I)$. The terms proportional to the quark masses in (2.3) induce new terms in the Lagrangian of $S$, that can be reshuffled into the coefficients of (2.2). For the first two terms one finds explicitly

$$
\lambda_{1} \rightarrow \lambda_{1}-8 F c_{1 m} B \hat{m}, \quad \stackrel{\circ}{m}_{S}^{2} \rightarrow m_{S}^{2}=\stackrel{\circ}{m}_{S}^{2}-16 c_{2 m} B \hat{m} .
$$

As a consequence the singlet field is brought out of its minimum in the chiral limit by terms proportional to $\hat{m}$. Hence, the direct consequence of the inclusion of non-vanishing quark masses results in a new contribution to the singlet-vacuum mixing. The new scalar field describing the first excitation with respect to the vacuum may be obtained by carrying out the following shift

$$
S \rightarrow S+F S_{0} \quad \text { with } \quad S_{0}=8 c_{1 m} \frac{B \hat{m}}{m_{S}^{2}}-\frac{\lambda_{1}}{m_{S}^{2} F} .
$$

For generic values of the LECs the shift (2.6) breaks chiral symmetry. Namely, if the original scalar field in (2.2) is a singlet under chiral symmetry, the scalar field after the shift (2.6) is not. This is so for any value of the parameters, except for those that fulfill $\lambda_{1}=\frac{c_{1 m} \dot{m}_{S}^{2} F}{2 c_{2 m}}$.

If we choose $\lambda_{1}$ as above, the shift becomes independent of the quark masses $\left(S_{0}=-c_{1 m} / 2 c_{2 m}\right)$, and hence the scalar field after the shift is still a scalar under chiral symmetry. However, the Lagrangian resulting from this field redefinition of $S$ is equivalent to choosing $\lambda_{1}=c_{1 m}=0$ in (2.2) and (2.3) on the original Lagrangian. If we impose to our original scalar field to be a singlet under chiral symmetry for any value of the external sources and not mix with the vacuum, then the only solution at tree level is $\lambda_{1}=c_{1 m}=0$. We shall adopt this option from now on.

\section{Two-point functions}

We are now in the position to perform a complete NLO analysis of the pion mass and decay constant, including the radiative correction due to the singlet field. We denote by $m_{\mathrm{PS}}^{2}$ and $F_{\mathrm{PS}}$ the pion mass and the pion decay constant respectively calculated at NLO, whereas we keep $m_{\pi}=2 B \hat{m}$ and $F$ for the same quantities at LO.

$$
\begin{aligned}
m_{\mathrm{PS}}^{2}= & 2 B \hat{m}-\frac{4 c_{1 d}^{2}}{F^{2}} \bar{J}\left(m_{\pi}^{2}, m_{S}^{2} ; m_{\pi}^{2}\right)\left(m_{S}^{2}-2 m_{\pi}^{2}\right)^{2}+\frac{4 m_{\pi}^{4}}{F^{2}}\left(\frac{\mu_{S}-\mu_{\pi}}{\Delta_{\pi S}}\right)\left(c_{1 d}^{2} m_{S}^{2}-4 c_{2 m} \Gamma_{1} \Delta_{\pi S}\right) \\
& +\frac{m_{\pi}^{4}}{16 \pi^{2} F^{2}} \gamma_{3} \bar{\ell}_{3}+\frac{m_{\pi}^{2} \stackrel{m}{S}_{S}^{2}}{8 \pi^{2} F^{2}} \Gamma_{1} \bar{Z}_{1}, \\
F_{\mathrm{PS}}= & F\left(1+\frac{2 c_{1 d}^{2}}{F^{2} m_{\pi}^{2}} \bar{J}\left(m_{\pi}^{2}, m_{S}^{2} ; m_{\pi}^{2}\right)\left(\frac{2 m_{\pi}^{2}-m_{S}^{2}}{4 m_{\pi}^{2}-m_{S}^{2}}\right)\left(14 m_{\pi}^{4}-15 m_{\pi}^{2} m_{S}^{2}+3 m_{S}^{4}\right)+\frac{c_{1 d}^{2}}{8 \pi^{2} F^{2}} \frac{\left(m_{S}^{2}-2 m_{\pi}^{2}\right)^{2}}{4 m_{\pi}^{2}-m_{S}^{2}}\right. \\
+ & \left.\frac{4 m_{\pi}^{2}}{F^{2}}\left(\frac{\mu_{\pi}-\mu_{S}}{\Delta_{\pi S}}\right)\left(\frac{c_{1 d}^{2}\left(m_{S}^{2}-2 m_{\pi}^{2}\right)^{2}}{\left(4 m_{\pi}^{2}-m_{S}^{2}\right)}+4 c_{2 m} \Gamma_{2} \Delta_{\pi S}\right)+\frac{m_{\pi}^{2}}{32 \pi^{2} F^{2}} \gamma_{4} \bar{\ell}_{4}+\frac{\grave{m}_{S}^{2}}{8 \pi^{2} F^{2}} \Gamma_{2} \bar{Z}_{2}\right)
\end{aligned}
$$




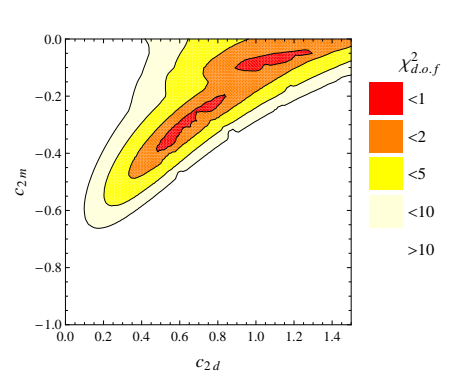

(a)

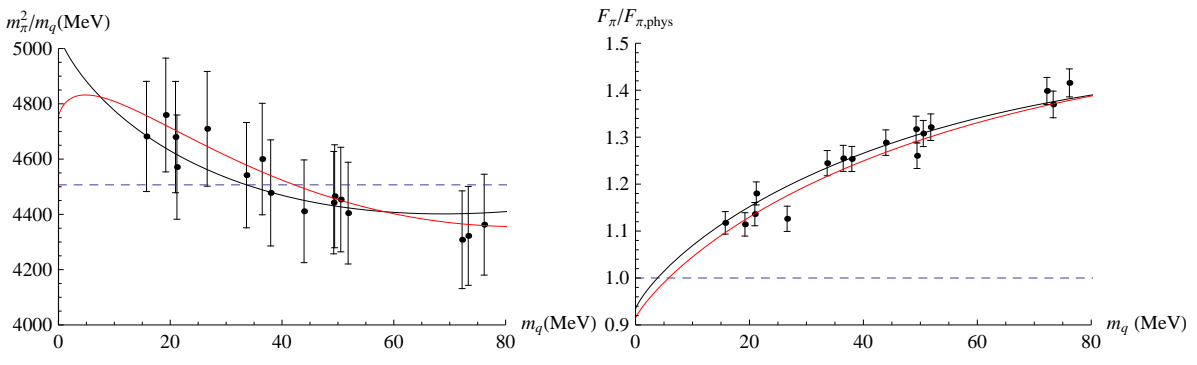

(b)

Figure 1: (a) $\chi_{\text {d.o.f }}^{2}$ swept over a $\left(c_{2 d}, c_{2 m}\right)$ grid corresponding to fits to NLO order expressions. (b) The best fits of the LO (dashed line), NLO $\chi \mathrm{PT}$ (black solid line) and $\chi \mathrm{PT}_{S}$ (red solid line) expressions. Note that the LO expression is the same for $\chi \mathrm{PT}$ and $\chi \mathrm{PT}_{S}$.

$\bar{J}$ and $\mu_{a}(a=\pi, S)$ are the standard one loop functions; $\Gamma_{1}, \Gamma_{2}, \gamma_{3}$ and $\gamma_{4}$ are the coefficients of the divergent terms of the LEC (see [10] for details). In addition we have used the scale independent quantities $\bar{\ell}_{i}$ and $\bar{Z}_{j}$, which are related to the finite part of the conterterms in (2.4).

The scalar mass at NLO, defined as the pole of the scalar field two-point function, reads as follows

$$
m_{S, \mathrm{NLO}}^{2}=m_{S}^{2}-\frac{m_{S}^{4}}{32 \pi^{2}} \Gamma_{f} \bar{f}_{2 p}-\frac{m_{\pi}^{4}}{32 \pi^{2}} \Delta_{1} \bar{d}_{1 m}-\frac{m_{\pi}^{2} m_{S}^{2}}{4 \pi^{2}} \Delta_{2} \bar{d}_{2 m}-\frac{6 c_{1 d}^{2}}{F^{2}} \bar{J}\left(m_{\pi}^{2}, m_{\pi}^{2} ; m_{S}^{2}\right)\left(m_{S}^{2}-2 m_{\pi}^{2}\right)^{2},
$$

where $\Gamma_{f}, \Delta_{1}$ and $\Delta_{2}$ are coefficients of the divergent terms of the LEC, and $\bar{f}_{2 p}, \bar{d}_{1 m}$ and $\bar{d}_{2 m}$ are scale independent quantities related to the counterterms [10].

The scalar decay width can be read from the $\bar{J}$ function

$$
\frac{\Gamma}{2}=\frac{3 c_{1 d}^{2}}{8 \pi F^{2} m_{S}} \sqrt{1-\frac{4 m_{\pi}^{2}}{m_{S}^{2}}}\left(m_{S}^{2}-2 m_{\pi}^{2}\right)^{2}
$$

Notice that it only depends on a single unknown LEC, $c_{1 d}$. Using the standard values for $F \sim F_{\pi}$ and $m_{\pi}$, and taking specific values for the mass and width of the sigma resonance from [4] we obtain from $c_{1 d}^{2}=0.457$.

\subsection{Matching with lattice data}

Lattice QCD offers a new arena for determining the LEC. Unlike physical experiments, lattice calculations use different unphysical quark masses, providing for each point what can be considered as an uncorrelated experimental datum with Gaussian errors. We will use the lattice data based on maximally $n_{f}=2$ twisted fermions to fit the LEC [6]. The fits are forced to reproduce the pion decay constant, the mass of the sigma resonance and its width at the physical point [10], $c_{1 d}^{2}$ is obtained from the previous section.

If we look at the contour level plot of the $\chi_{\text {d.o.f }}^{2}$ corresponding to the $\left(c_{2 d}, c_{2 m}\right)$ region scanned, shown in Fig. 1, we can see regions of parameter sets with $\chi_{\text {d.o.f }}^{2}$ smaller than one. Thus any parameter set on those regions has to be considered a valid solution. Keeping this in mind, the following are the results for the best fit obtained $\left(\chi_{\text {d.o.f }}^{2}=16.7 / 26\right)$, which have been used for Fig. 1.b .

$$
\begin{aligned}
& B=1680.5 \mathrm{MeV}, \quad F=101.2 \mathrm{MeV}, \quad \stackrel{\circ}{m}_{S}=426 \mathrm{MeV}, \\
& c_{2 d}=1.21, \quad c_{2 m}=-0.083, \quad \ell_{3}^{r}=-1.12 \times 10^{-3}, \quad \ell_{4}^{r}=6.94 \times 10^{-3} .
\end{aligned}
$$




\section{S-wave $\pi-\pi$ scattering lengths}

Let us next consider $\pi-\pi$ scattering. Due to the presence of a novel contribution coming with a scalar particle in the intermediate state, we expect a LO correction to the $\chi$ PT results. Let us now turn to the evaluation of the scattering lengths. Their explicit expressions at LO are given by

$$
a_{0}^{0}=\frac{m_{\pi}^{2}}{\pi F^{2}}\left(\frac{7}{32}-\frac{3}{2} \frac{m_{\pi}^{2}}{4 m_{\pi}^{2}-m_{S}^{2}} c_{1 d}^{2}+\frac{m_{\pi}^{2}}{m_{S}^{2}} c_{1 d}^{2}\right), \quad a_{0}^{2}=-\frac{m_{\pi}^{2}}{\pi F^{2}}\left(\frac{1}{16}-\frac{m_{\pi}^{2}}{m_{S}^{2}} c_{1 d}^{2}\right) .
$$

In the decoupling limit $\left(\stackrel{\circ}{m}_{S}^{2} \gg m_{\pi}^{2}, p^{2}\right)$ we expect to recover the $\chi$ PT expression. It can be shown that the new contribution due to the scalar field gives a matching contribution to the $l_{1} \chi \mathrm{PT}$ counterterm. Since the new contribution is LO and the $l_{1} \chi \mathrm{PT}$ counterterm is NLO, we expect this matching contribution to be large. This indicates that a large negative value is expected for $\ell_{1}$, and consequently that NLO contributions to $\pi-\pi$ scattering are going to be large in order to reproduce the standard values for $l_{1} \chi \mathrm{PT}$ counterterm in the decoupling limit [10].

\subsection{Matching with lattice data}

The available lattice results for the S-wave scattering lengths use relatively large pion masses, which makes chiral extrapolations less reliable. In fact, until recently only calculations of $a_{0}^{2}$ were available, and the only existing calculation of both $a_{0}^{2}$ and $a_{0}^{0}$ neglects the disconnected contributions to the latter [7]. Nevertheless we shall use lattice data of the last reference in order to get a feeling on how $\chi \mathrm{PT}_{S}$ performs with respect to the $\mathrm{S}$-wave scattering lengths.

The S-wave scattering lengths of $\chi \mathrm{PT}_{S}$ at $\mathrm{LO}$ are fixed once we input the mass and the width of the sigma resonance in addition to the pion mass and decay constant. Their evolution with the light quark masses is given by that of the pion mass and the LEC $c_{2 m}$. By making a combined fit to $a_{0}^{2}$ and $a_{0}^{0}$ we obtain the dashed red line in Fig. 2. We observed that for $a_{0}^{0} \chi \mathrm{PT}_{S}$ provides a better description of data than LO $\chi \mathrm{PT}$ (dashed black line), but for $a_{0}^{2}$ a much worse one. As argued in section 4 , large NLO corrections due to $\ell_{1}$ are expected. We may estimated them by just adding its contribution to LO expression. If we fit $\ell_{1}$, we obtain the dashed red line in Fig. 2, and the following numbers

$$
a_{0}^{0}=0.210 \quad, \quad a_{0}^{2}=-0.0296 \quad, \quad c_{2 m}=-0.443 \quad, \quad \bar{\ell}_{1} \equiv 96 \pi^{2} \ell_{1}=-16.9 .
$$

Note that we get a large negative number for $\bar{\ell}_{1}$, consistent with the expectations. We see that the description of both scattering lengths improves considerably, the quality of $a_{0}^{0}$ being comparable to that of NLO $\chi$ PT (black solid line). The values of the LEC of $\chi$ PT delivered by the fit are quite different from the standard values.

The results above encourage us to attempt an extraction of the sigma resonance parameters from the lattice data.

$$
\begin{array}{lll}
c_{2 m}=-0.228 & , \quad \bar{\ell}_{1}=-10.9 \quad, \quad c_{1 d}^{2}=0.304 \quad, \quad \stackrel{\circ}{S}_{S}=483 \mathrm{MeV}, \\
m_{S}=486 \mathrm{MeV} & , \quad \frac{\Gamma}{2}=236 \mathrm{MeV} \quad, \quad a_{0}^{0}=0.177 \quad, \quad a_{0}^{2}=-0.0361 .
\end{array}
$$

The numbers above are quite reasonable for a $\mathrm{LO}$ approximation augmented by $\ell_{1}$, even more if one takes into account that the lattice data is at relatively large pion masses. It shows that our approach may eventually allow for a precise extraction of the sigma resonance parameters from lattice QCD. 


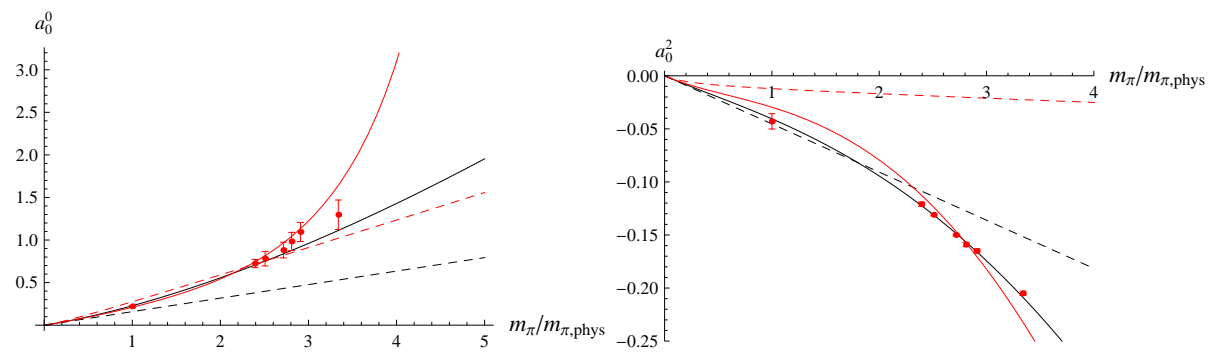

Figure 2: The best fits of the LO $\chi \mathrm{PT}$ (black dashed line), NLO $\chi \mathrm{PT}$ (black solid line), LO $\chi \mathrm{PT}_{S}$ (red dashed line) and LO $\chi \mathrm{PT}_{S}$ augmented by the operator proportional to $\ell_{1}$ (red solid line). Red dots are lattice data from [7].

\section{Conclusions}

We have considered the possibility that the spectrum of QCD in the chiral limit contains an isosinglet scalar with a mass much lower than the typical hadronic scale $\Lambda_{\chi}$, and have constructed the corresponding effective theory that includes it together with the standard pseudo-Goldstone bosons, $\chi \mathrm{PT}_{S}$. This has consequences concerning the dependence of physical observables on the light quark masses, which have been shown to be compatible with current lattice data.

\section{Acknowledgments}

We have been supported by the CPAN CSD2007-00042 Consolider-Ingenio 2010 program (Spain), the 2009SGR502 CUR grant (Catalonia) and by the FPA2010-16963 project (Spain). JT acknowledges a MEC FPU fellowship (Spain).

\section{References}

[1] J. Gasser and H. Leutwyler, Annals Phys. 158, 142 (1984).

[2] A. Dobado and J. R. Pelaez, Phys. Rev. D 56, 3057 (1997) [arXiv:hep-ph/9604416].

[3] J. A. Oller and E. Oset, Nucl. Phys. A 620, 438 (1997) [Erratum-ibid. A 652, 407 (1999)] [arXiv:hep-ph/9702314].

[4] I. Caprini, G. Colangelo and H. Leutwyler, Phys. Rev. Lett. 96, 132001 (2006) [arXiv:hep-ph/0512364].

[5] R. Garcia-Martin, R. Kaminski, J. R. Pelaez and J. Ruiz de Elvira, Phys. Rev. Lett. 107, 072001 (2011) [arXiv:1107.1635 [hep-ph]].

[6] R. Baron et al. [ETM Collaboration], JHEP 1008, 097 (2010) [arXiv:0911.5061 [hep-lat]].

[7] Z. Fu, Commun. Theor. Phys. 57, 78 (2012) [arXiv:1110.3918 [hep-lat]].

[8] M. Luscher and P. Weisz, Nucl. Phys. B 290, 25 (1987).

[9] J. Frohlich, Nucl. Phys. B 200, 281 (1982).

[10] J. Soto, P. Talavera and J. Tarrus, Nucl. Phys. B 866 (2013) 270 [arXiv:1110.6156 [hep-ph]]. 